\title{
MicroRNA-122-5p Aggravates Angiotensin II-Mediated Myocardial Fibrosis and Dysfunction in Hypertensive Rats by Regulating the Elabela/Apelin-APJ and ACE2-GDF15-Porimin Signaling
}

\author{
Jiawei Song ${ }^{1,2,3}$. Zhenzhou Zhang ${ }^{1,2} \cdot$ Zhaojie Dong $^{1,2} \cdot$ Xinming Liu ${ }^{1,2} \cdot$ Ying Liu $^{1,2,3} \cdot$ Xueting $\mathrm{Li}^{1,2} \cdot$ Yingle Xu $^{4}$. \\ Ying Guo ${ }^{5} \cdot$ Ning Wang ${ }^{5} \cdot$ Miwen Zhang ${ }^{1,2} \cdot$ Yihang Chen ${ }^{1,2} \cdot$ Haiyan Jin $^{4} \cdot$ Jiuchang Zhong ${ }^{1,2,3}$ (D)
}

Received: 6 December 2021 / Accepted: 31 January 2022 / Published online: 16 February 2022

(c) The Author(s), under exclusive licence to Springer Science+Business Media, LLC, part of Springer Nature 2022

\begin{abstract}
Hypertension is the leading risk factor for cardiovascular disorders. This study aimed to explore roles of microRNA (miR)$122-5 \mathrm{p}$ in hypertension. Angiotensin II (Ang II; $1.5 \mathrm{mg} / \mathrm{kg} /$ day) with an osmotic minipump was used to induce hypertensive rats pretreated by rAAV-miR-122-5p or rAAV-GFP, respectively. Notably, Ang II infusion caused marked increases in myocardial fibrosis, inflammation, oncosis, and oxidant injury in rats, which were aggravated by rAAV-miR-122-5p. RAAVmiR-122-5p exacerbated Ang II-mediated cardiac dysfunction and structural injury in hypertensive rats, with downregulated levels of apelin, elabela, ACE2, and GDF15, as well as upregulated expression of porimin and CTGF. In cultured rat cardiac fibroblasts, Ang II contributed to augmentation of cellular oncosis, migration, inflammation, and oxidative stress, with reduction of apelin, elabela, ACE2, and GDF15 levels, which were rescued by miR-122 inhibitor. In summary, miR-122-5p exacerbates myocardial fibrosis and dysfunction in hypertensive rats by modulating the elabela/apelin-ACE2-GDF15 signaling. MiR-122-5p has potential therapeutic significance for hypertension and hypertensive cardiac injury.
\end{abstract}

Keywords miR-122-5p $\cdot$ Elabela $\cdot$ Oncosis $\cdot$ Myocardial fibrosis $\cdot$ Hypertension

Editor-in-Chief Enrique Lara-Pezzi oversaw the review of this article.

Jiawei Song and Zhenzhou Zhang contributed equally to this work

Haiyan Jin

hyjin603@163.com

$\triangle$ Jiuchang Zhong

jczhong@sina.com

1 Heart Center and Beijing Key Laboratory of Hypertension, Beijing Chaoyang Hospital, Capital Medical University, Beijing, China

2 Department of Cardiology, Beijing Chaoyang Hospital, Capital Medical University, Beijing, China

3 Medical Research Center, Beijing Institute of Respiratory Medicine and Beijing Chaoyang Hospital, Capital Medical University, Beijing, China

4 Department of Psychiatry \&, School of Medicine, Shanghai Institute of HypertensionRuijin HospitalShanghai Jiao Tong University, Shanghai, China

5 Department of Geratology, Beijing Tongren Hospital, Capital Medical University, Beijing, China

\section{Introduction}

Hypertension is the leading modifiable risk factor for cardiovascular diseases [1]. Left ventricular remodeling is frequently seen in hypertensive subjects and has been considered an adaptive response to mechanical and nonmechanical stimuli imposed by systemic hypertension [2]. Angiotensin II (Ang II), the major effecter in the renin-angiotensin system (RAS), has a critical role in the maintenance of blood pressure levels [2]. Although increasing evidences indicate that abnormal activation of the RAS is responsible for aberrant cell death, oxidative stress, inflammation, and fibrosis during pathological cardiac remodeling, the underlying genes and pathways during these pathological processes remain largely unknown. Further investigations of biomarkers and therapeutic targets related to the pathogenesis of hypertensive heart injury are required.

Growth differentiation factor 15 (GDF15), a stressresponsive cytokine, is highly expressed in cardiovascular system and has been identified as a biomarker of response to treatment and prognosis in cardiovascular diseases [3]. A significant positive correlation was found between Ang II and GDF15 levels in hypertensive patients [4], whereas the regulatory mechanisms 


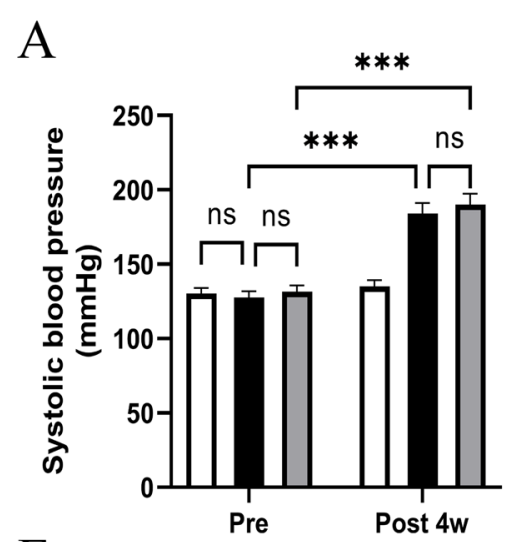

$\mathrm{B}_{\text {Apelin }}$

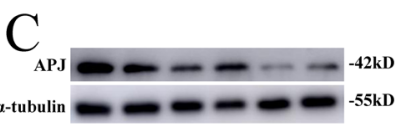

D
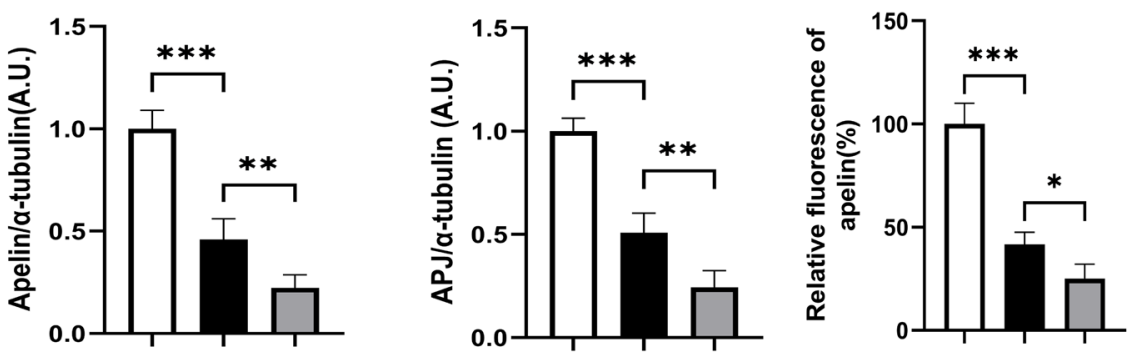

E
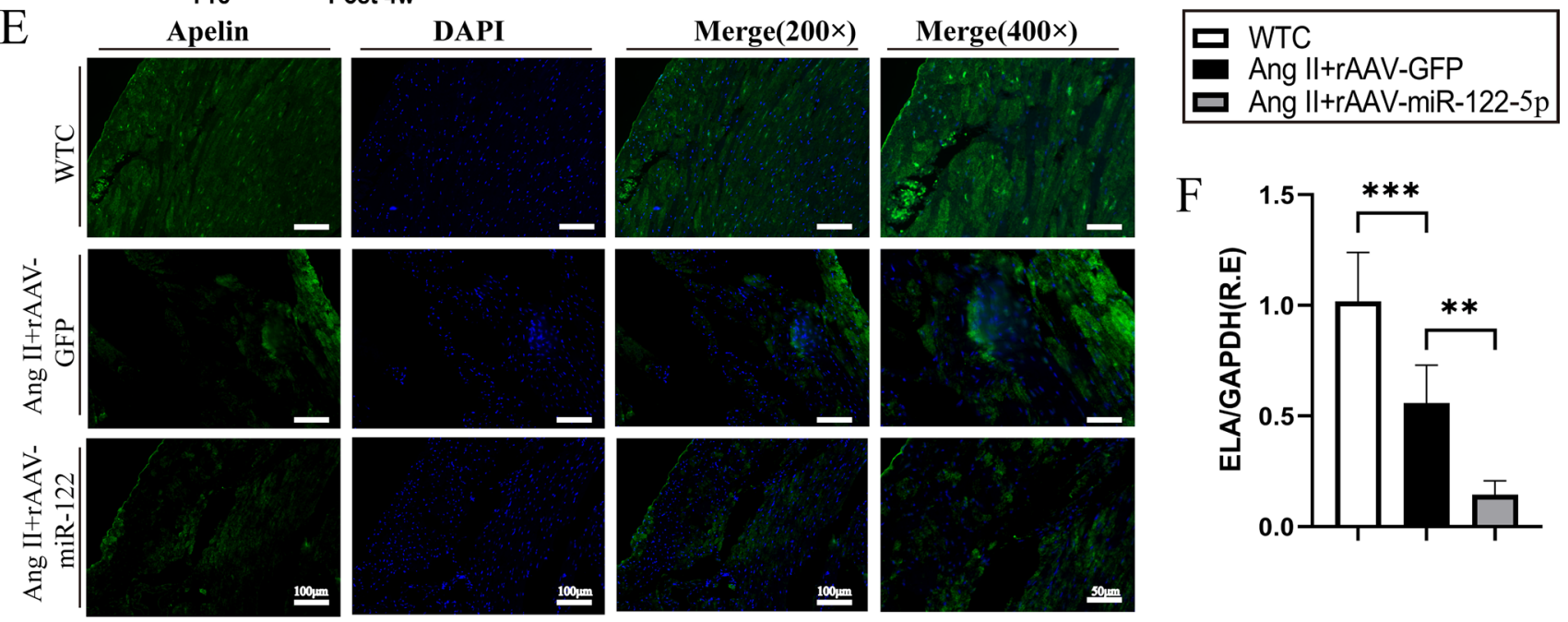

G

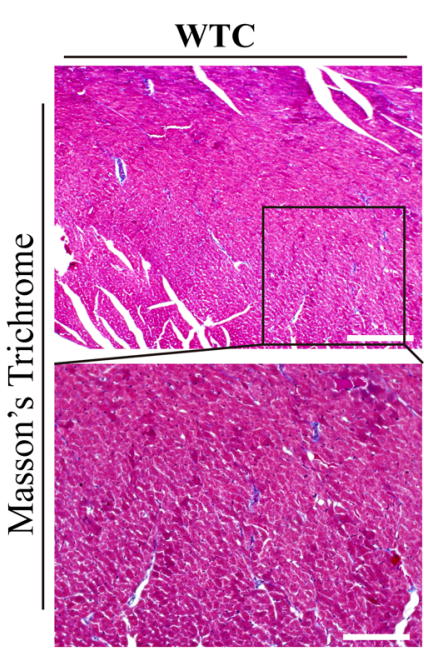

Ang II+rAAV-GFP

Ang II+rAAV-miR-122-5p
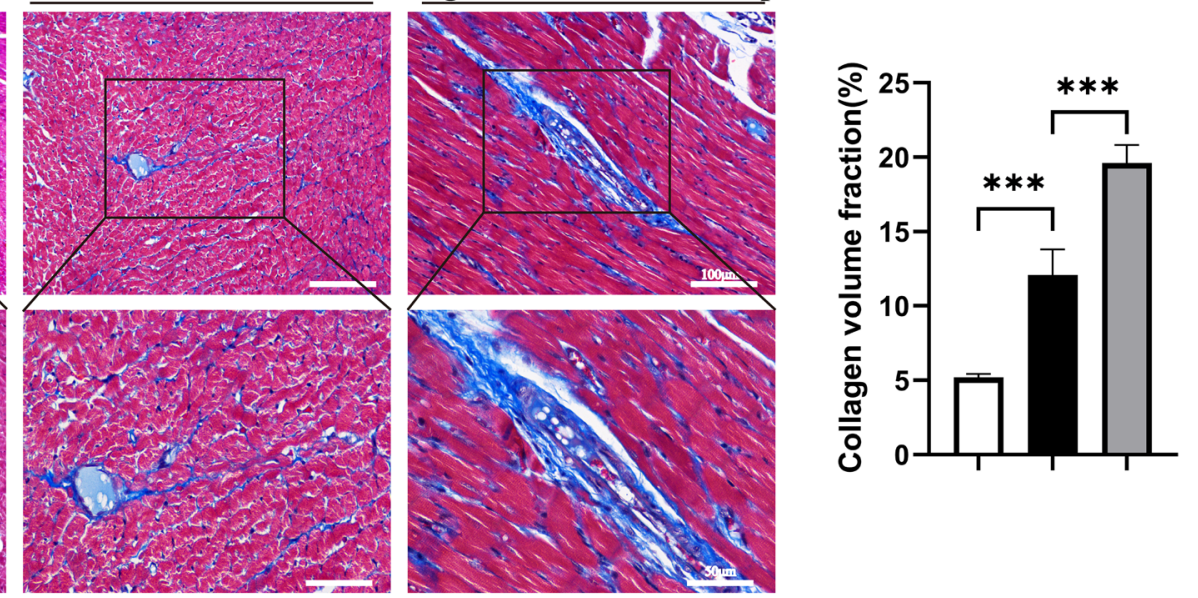

I
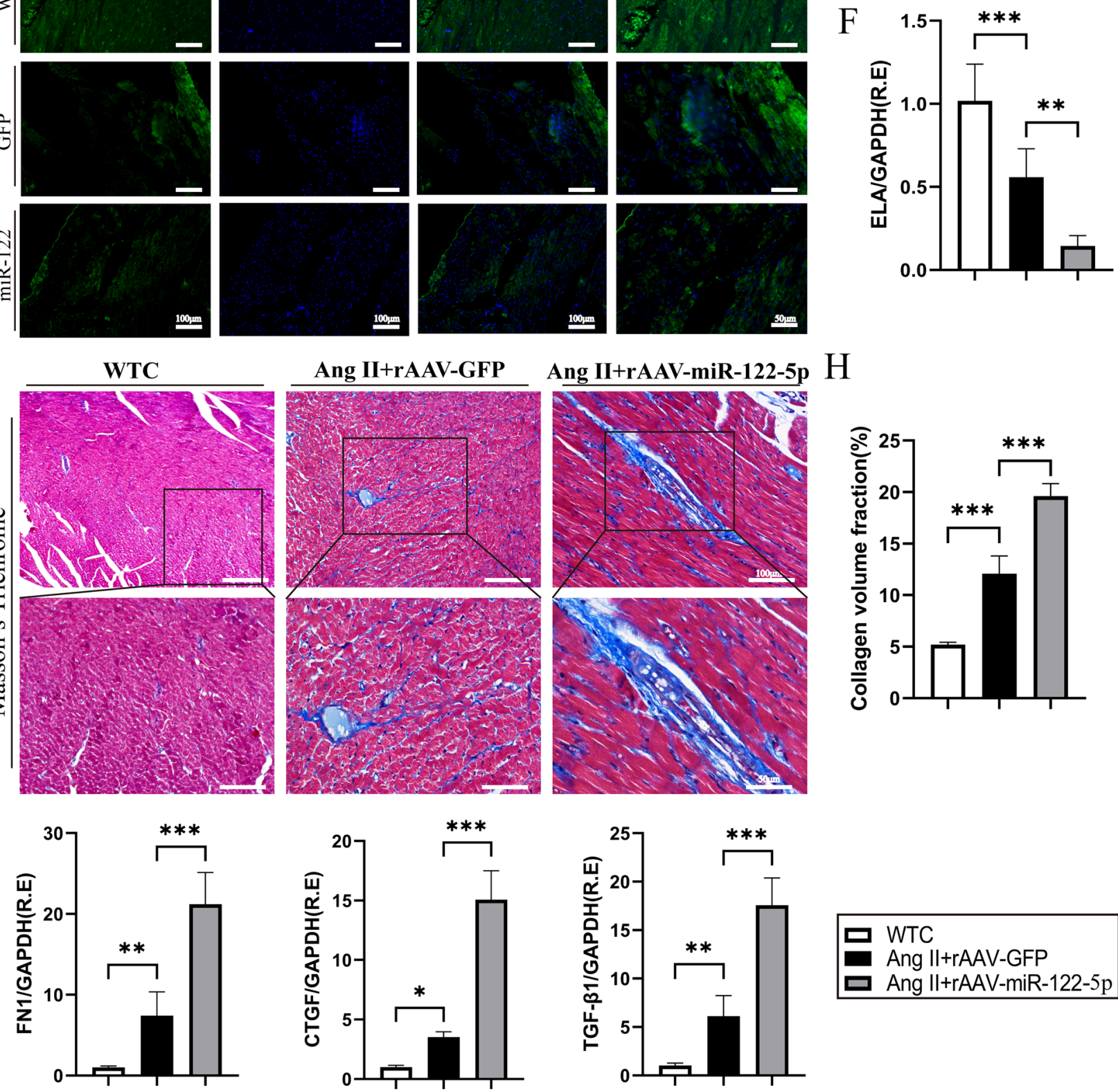

$\mathrm{H}$ 
४Fig. 1 Treatment with rAAV-miR-122-5p promoted cardiac fibrosis in hypertensive rats with downregulated levels of apelin, APJ, and elabela. A Infusion of Ang II for 4 weeks resulted in elevated SBP in rats, but treatment with rAAV-miR-122-5p did not affect SBP levels in hypertensive rats. B-F MiR-122-5p overexpression promoted Ang II-mediated downregulation of apelin $(\mathbf{B}, \mathbf{D}, \mathbf{E})$, APJ $(\mathbf{C})$, and ELA $(\mathbf{F})$ in hearts of rats. $\mathbf{G}-\mathbf{K}$ Masson's trichrome staining and quantitative reverse transcription-PCR revealed that miR-122-5p overexpression exacerbated cardiac fibrosis $(\mathbf{G}, \mathbf{H})$ in hypertensive rats, along with increased mRNA expression of pro-fibrotic factors, such as FN1 (I), CTGF (I), and TGF- $\beta 1$ (I). A.U., arbitrary unit; R.E., relative expression; WTC, wild-type control; rAAV, recombinant adeno-associated virus; GFP, green fluorescent protein; SBP, systolic blood pressure; FN1, fibronectin 1; CTGF, connective tissue growth factor; TGF- $\beta 1$, transforming growth factor- $\beta 1$; APJ, apelin receptor; Ang II, angiotensin II. $n=6-8$ for each group except for $\mathbf{G}-$ H where $n=4$. $* P<0.05, * * P<0.01, * * * P<0.001$

of GDF15 in hypertensive heart remodeling remained elusive. Recently, the Yes-associated protein (YAP), an evolutionarily and functionally conserved regulator of organ size and growth, has drawn great attention for its tremendous potential for developing therapeutic strategies for intractable cardiovascular diseases [5]. The YAP pathway is an important signaling branch downstream of Ang II type 1 receptor in cardiac fibroblasts (CFs) mechano-transduction [6]. YAP has been shown to be activated in CFs in response to Ang II, and blockade of fibroblast YAP attenuated cardiac fibrosis and dysfunction [7]. Both GDF15 and YAP are involved in Ang II-mediated cardiac fibrosis, but the underlying mechanisms need to be further explored.

MiRNAs (miRs) function as small and high conserved noncoding RNAs, negatively regulating translation or promote degradation of mRNA by targeting the 3' non-coding region [8]. Dysregulation of miRs is increasingly being linked to cardiovascular diseases, of which miR-122-5p has drawn significant attention [9]. MiR-122-5p has been proved to participate in the occurrence and development of hypertension, promoting cardiac hypertrophy and cardiomyocyte apoptosis [8]. However, the role and mechanisms of miR-122-5p in hypertensive heart disease have not been fully elucidated. We have reported that miR-122-5p aggravated Ang II-mediated apoptosis and fibrosis in rat aortic adventitial fibroblasts via modulating the SIRT6-elabela-angiotensin-converting enzyme 2 (ACE2) signaling [10]. Additionally, apelin, which is a positive regulator of ACE2 and negative regulator of Ang II-mediated adverse myocardial remodeling and dysfunction [11], ameliorates aortic adventitial remodeling and fibrosis in rats via suppression of miR-122-5p [12]. Previously, apelin was thought to be the only ligand for apelin receptor (APJ), while elabela (ELA) was identified as a second endogenous ligand for the APJ. The ELA/apelin-APJ axis exerts essential roles in regulating blood pressure levels and cardiovascular dysfunction during hypertension by counterbalancing the action of RAS [13] implying an interaction between miR-122-5p and the ELA/apelin-APJ axis However, the exact mechanisms and underlying pathways of miR-122-5p in hypertensive cardiac injury remain to be identified. In the present study, we discovered a previously unrecognized role of miR-122-5p in the development of cardiac fibrosis and dysfunction in the context of hypertension, highlighting a novel molecular mechanism of miR122-5p during hypertension with potential therapeutic relevance.

\section{Materials and Methods}

\section{Experimental Animals}

Sprague-Dawley (SD) rats (10-11 weeks) were obtained from Shanghai SLAC Laboratory Animal Co., LTD (Shanghai, China). The recombinant AAV-miR-122-5p was purchased from Shanghai Genechem Co., Ltd. (Shanghai, China). Animals were randomly divided into the following three groups: wild-type control (WTC) group, Ang II + rAAV-GFP group, and Ang II + rAAV-miR-122-5p group. The Ang II-infused rats were pre-treated by tail intravenous injection of rAAV-miR-122-5p or rAAV-GFP at the dose of $1 \times 10^{11} \mathrm{v} . \mathrm{g}$.. After injection for 4 weeks, the rats were randomized to deliver either Ang II $(1.5 \mathrm{mg} / \mathrm{kg} /$ day; Sigma-Aldrich, MO, USA) with an osmotic minipump (Alzet model 2004, MA, USA) for another 4 weeks. Systolic blood pressure (SBP) levels of rats were measured non-invasively using the tail-cuff method. Then, after 4-week Ang II infusion, heart function was measured noninvasively by transthoracic echocardiography using a VEVO 770 highresolution imaging system (Canadian VisualSonics) and a 30-MHz sensor (rmv-707b; Canadian VisualSonics). Then the rats were weighed and anesthetized, hearts were immediately removed and weighed, and tissue analysis was carried out.

\section{Histological Analysis, Apelin, GDF15, and Porimin Immunofluorescence Assay}

Myocardial fibrosis was measured by Masson's trichrome according to the manufacturer's instructions as previously described [14]. Paraffin-embedded heart tissue slices were deparaffinized and rehydrated with $100 \%, 95 \%$, and $70 \%$ ethanol, followed by staining by hematoxylin method and rinsed with distilled water. Then, slices were immersed in Masson ponceau acid solution for $10 \mathrm{~min}$, then differentiated with $1 \%$ molybdenum phosphate acid for $3 \mathrm{~min}$. Then, 
A
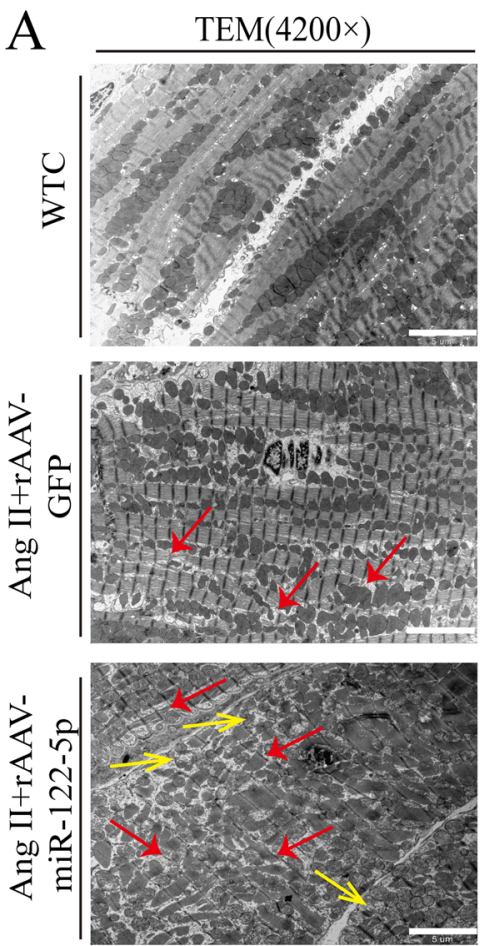

$\operatorname{TEM}(13500 \times)$
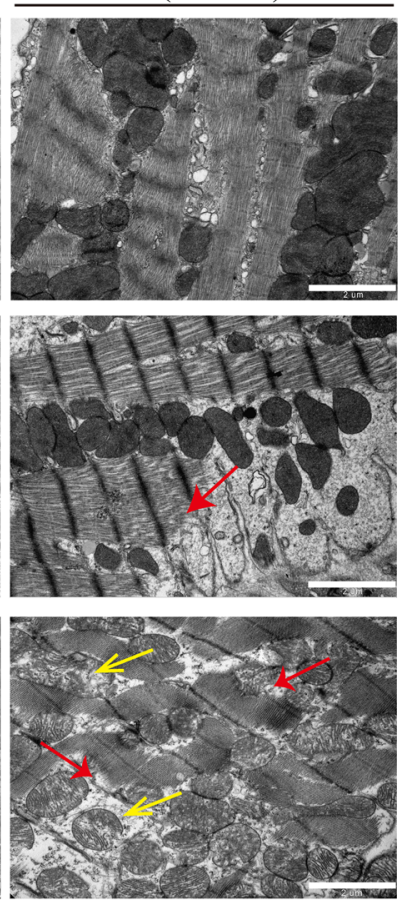

B Echocardiography
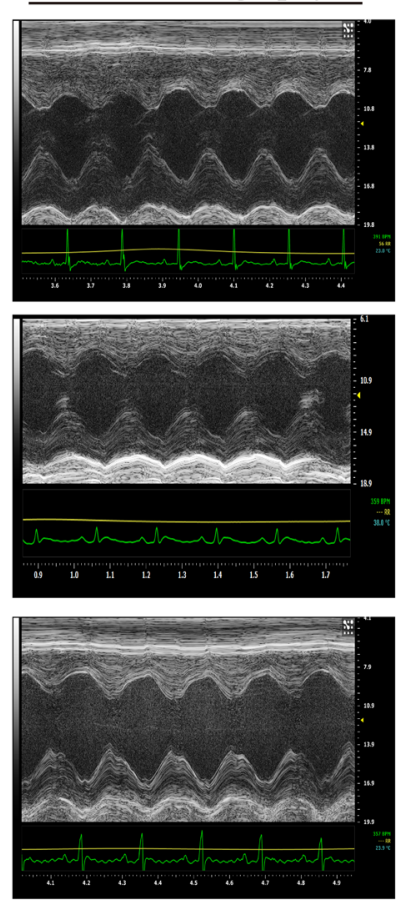

$\operatorname{Merge}(100 \times)$
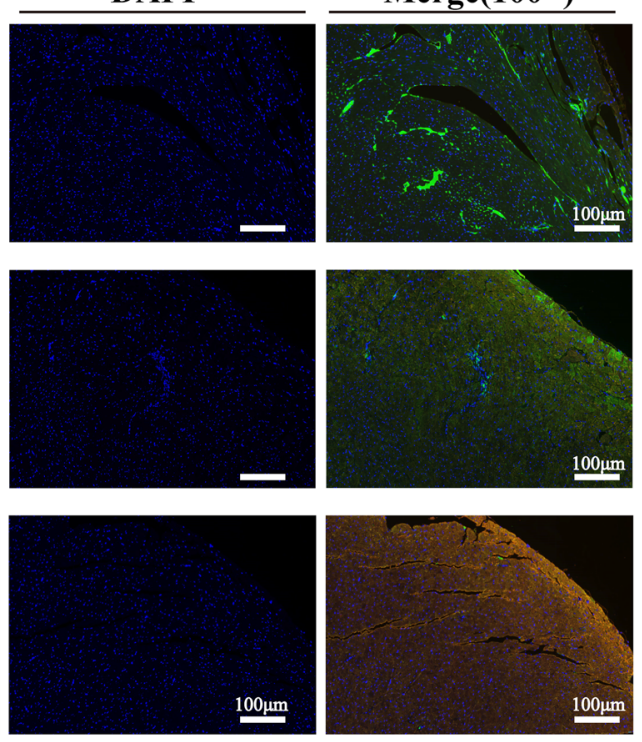

G
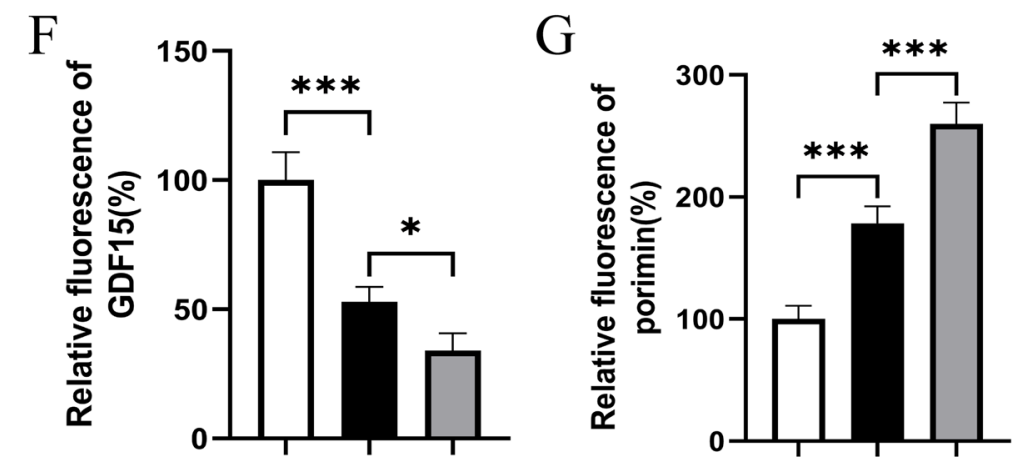

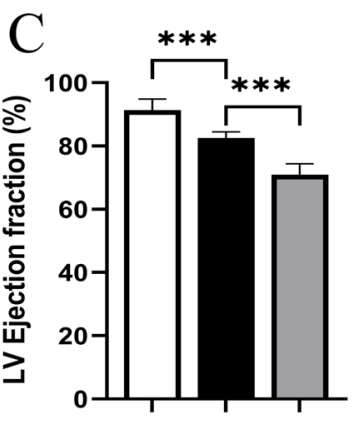

$\mathrm{D}$

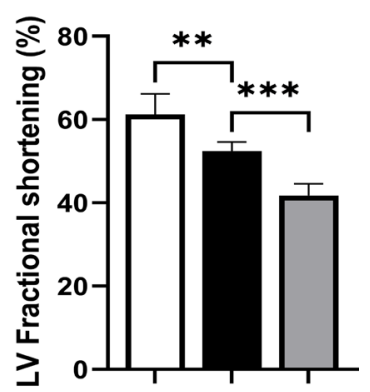

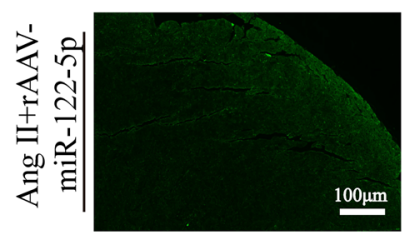
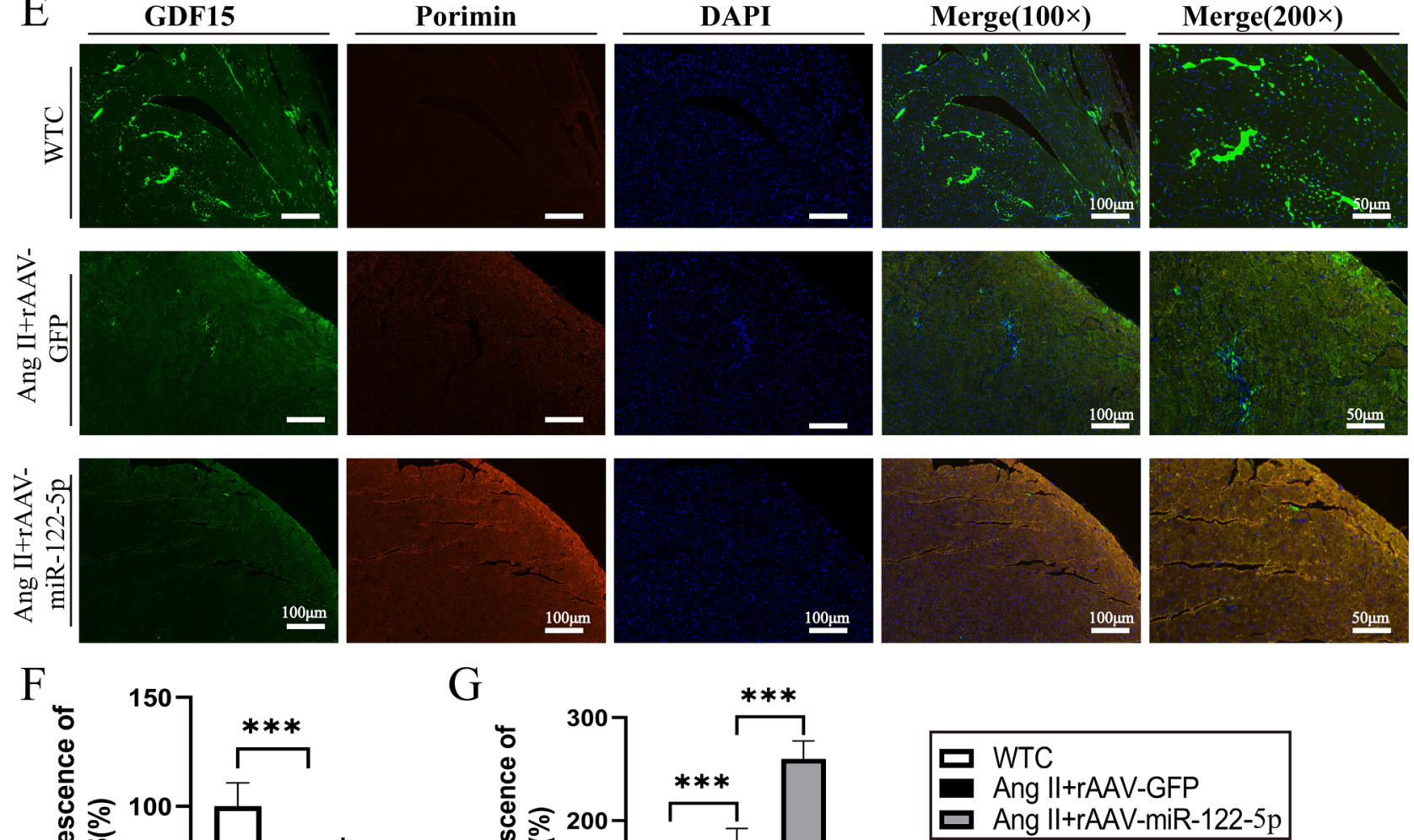
4Fig. 2 MiR-122-5p overexpression exacerbated cardiac ultrastructural injury and dysfunction in hypertensive rats, along with reduced GDF15 expression and increased porimin levels. A Overexpression of miR-122-5p exacerbated cardiac ultrastructural injury in hypertensive rats, which characterized by myofilament break (red arrow) and mitochondrial cristae rarefication (yellow arrow). B-D MiR-122-5p promoted Ang II-induced cardiac dysfunction (B) in rats, as evidenced by reduced left ventricular ejection fraction $(\mathbf{C})$ and fractional shortening (D). E-G Immunofluorescent staining of heart tissue showed the expression levels of $\mathrm{GDF} 15(\mathbf{E}, \mathbf{F})$ and porimin $(\mathbf{E}, \mathbf{G})$ in each group. WTC, wild-type control; rAAV, recombinant adeno-associated virus; GDF15, growth differentiation factor 15; GFP, green fluorescent protein; Ang II, angiotensin II. $n=6$ for each group except for $\mathbf{E}-\mathbf{G}$ where $n=4$. $* * P<0.01, * * * P<0.001$

the sections were directly transferred to aniline blue dyeing solution for $6 \mathrm{~min}$; afterwards, sections were rinsed briefly in distilled water and differentiated in $1 \%$ glacial acetic acid. Finally, sections were dehydrated $95 \%$ and $100 \%$ ethanol and then in xylene for $5 \mathrm{~min}$ before sealing with neutral gum. Visualizations of sections were then observed under a light microscope.

APLN (GenBank accession No.: NM_017413.5) and APLNR (GenBank accession No.: NM_031349.2) were identified as targets of miR-122-5p by bioinformatics tools Microcosm Targets and microrna.org (Fig. S1A). For heart tissue immunofluorescence assay, the pretreated tissue slices were processed for immunostaining of $1 \mathrm{~h}$ blocking in $10 \%$ BSA plus $0.2 \%$ Triton X-100 at room temperature, overnight incubation with primary antibodies against apelin (Cell Signaling Technology, MA, USA), GDF15 (Santa Cruz, CA, USA), and porimin (Santa Cruz, CA, USA) at $4{ }^{\circ} \mathrm{C}$, and for $1 \mathrm{~h}$ at room temperature incubation with appropriate secondary antibodies (Proteintech, IL, USA). Finally, the nuclei were stained with flourished mounting medium with DAPI (Invitrogen, CA, USA) intended for mounting slides. Fluorescent images were captured by an Olympus IX51 microscope (Olympus, Tokyo).

\section{Transmission Electron Microscope Analysis}

Transmission electron microscope (TEM) analyses of heart samples and CFs of rats were carried out as described previously [14]. The samples fixed with $2.5 \%$ glutaraldehyde were chopped into $0.1-\mathrm{cm}^{3}$ pieces. Followed by postfixation with $1 \%$ osmium tetroxide for $1 \mathrm{~h}$ and stained with $2 \%$ aqueous uranyl acetate, samples were then dehydrated in a graded series of ethanol. After infiltration and polymerization, ultrathin sections were cut and flattened with xylene vapor, then collected on nickel grids. Then, the images were observed under a transmission electron microscope (Philips, CM120, Amsterdam, Holland).
Isolation and Culture of Primary Neonatal Rat CFs and Cardiomyocytes (CMs)

Primary neonatal rat CFs were isolated from hearts of 2- to 3-day-old SD rats as previously described [14]. Cells were cultured in DMEM containing 1\% penicillin and streptomycin and $10 \%$ fetal bovine serum (FBS) in a $5 \% \mathrm{CO}_{2}$ atmosphere at $37{ }^{\circ} \mathrm{C}$. Primary cultured CFs were identified by primary antibodies against $\alpha$-smooth muscle actin $(\alpha$-SMA; Cell Signaling Technology, MA, USA) and vimentin (Proteintech, IL, USA) (Fig. S2). CFs from passages 3 to 5 were replaced with the serum-free medium for $24 \mathrm{~h}$ when the cell density reaches $70 \%$, and treated with the absence and presence of apelin-13 (100 nM; TOCRIS, Bristol, UK), miR-122 inhibitor (50 nM; GenePharma, Shanghai), miRNA negative control (50 $\mathrm{nM})$, ELA (100 nM; MedChemExpress, NJ, USA), recombinant human ACE2 (rhACE2, $0.1 \mathrm{ng} / \mu \mathrm{l}$; MedChemExpress, NJ, USA), and N-acetyl-L-cysteine (NAC, $5 \mathrm{mM}$; Sigma-Aldrich, MO, USA), respectively. The abovementioned agents were added to CFs for $30 \mathrm{~min}$ prior to $24 \mathrm{~h}$ exposure of Ang II ( $1 \mu \mathrm{M}$; Sigma-Aldrich, MO, USA). The treated cells were then subjected to in vitro experiments. The primary CMs were treated with Ang II for $24 \mathrm{~h}$ and then subjected to quantitative reverse transcription PCR analysis.

\section{Quantitative Reverse Transcription PCR}

Total RNA was extracted from heart tissues or cells using the Trizol (Sigma-Aldrich, MO, USA). Then, RNA was reverse transcribed to cDNA according to the manufacturer's protocols (Takara, Japan; Tiangen, China). The primers for apelin, elabela, interleukin- $1 \beta$ (IL-1 $\beta$ ), interleukin-6 (IL-6), monocyte chemoattractant protein-1 (MCP-1), fibronectin1 (FN1), connective tissue growth factor (CTGF), transforming growth factor- $\beta 1$ (TGF- $\beta 1$ ), glyceraldehyde-3-phosphate dehydrogenase (GAPDH), miR-122-5p, and U6 are listed in Supplemental Table S1. The mRNA expression levels were determined on the Roche LightCycler 480 System using SYBR Premix Ex Taq Kit (Takara, Japan) and miRNA SYBR Green qPCR Mix (Tiangen, China).

\section{Western Blotting Analysis}

The heart samples and CFs cells were homogenized in lysis buffer (Beyotime, Shanghai, China) containing $1 \times$ protease inhibitors (Beyotime, Shanghai, China). After cryogenic centrifuge, the supernatant was collected. The protein concentration was measured by the Enhanced BCA Protein 

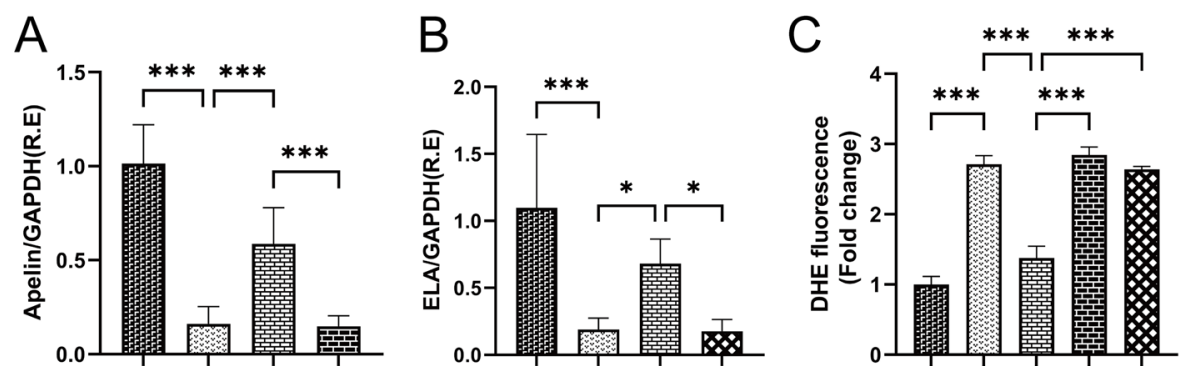

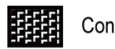
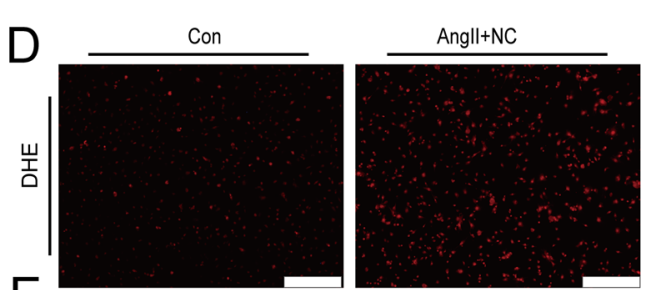

Angll+miR-122 inhibitor

Angll+miR-122 inhibitor+ Angll+NC

E
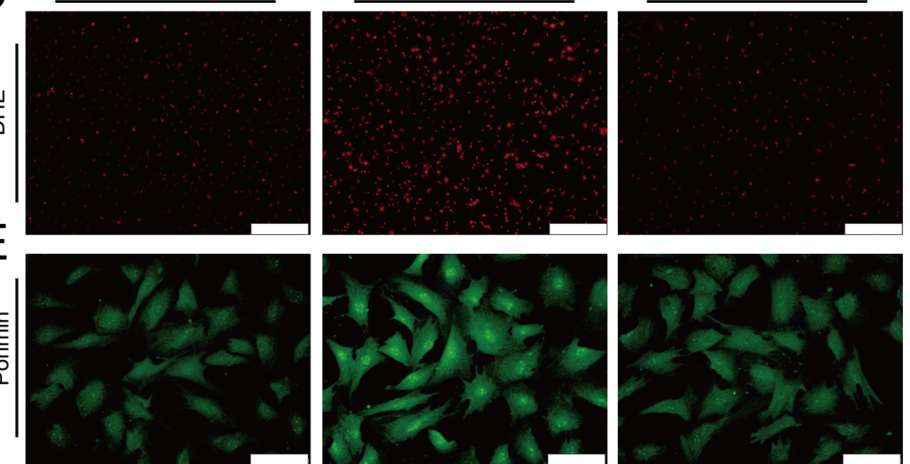

Apelin SIRNA

Angll+miR-122 inhibitor
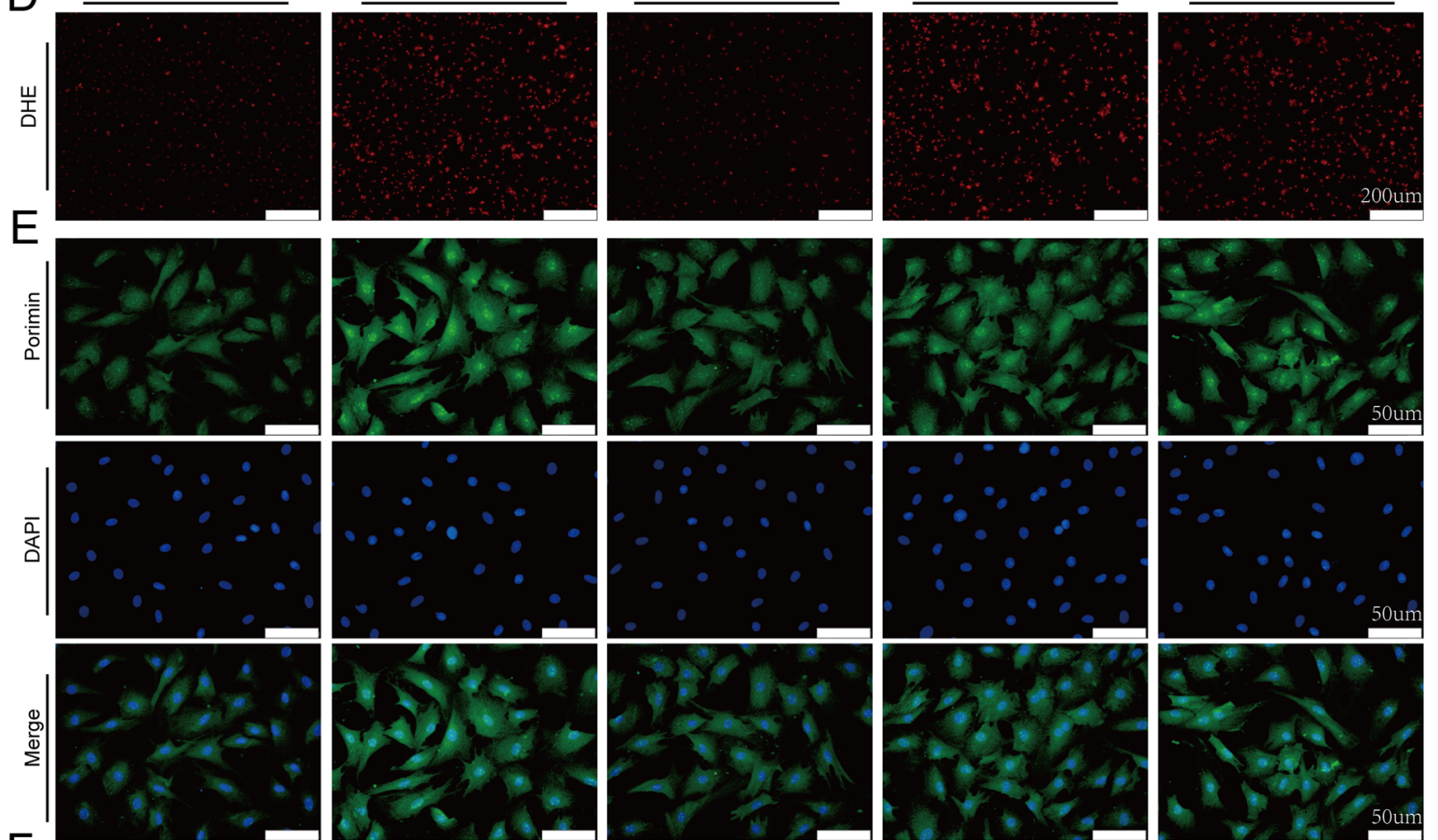

$\mathrm{F}$
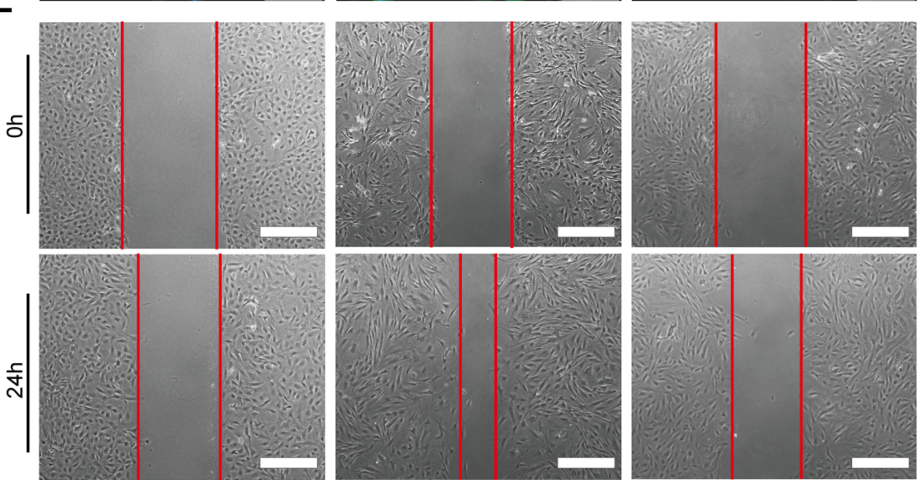

G
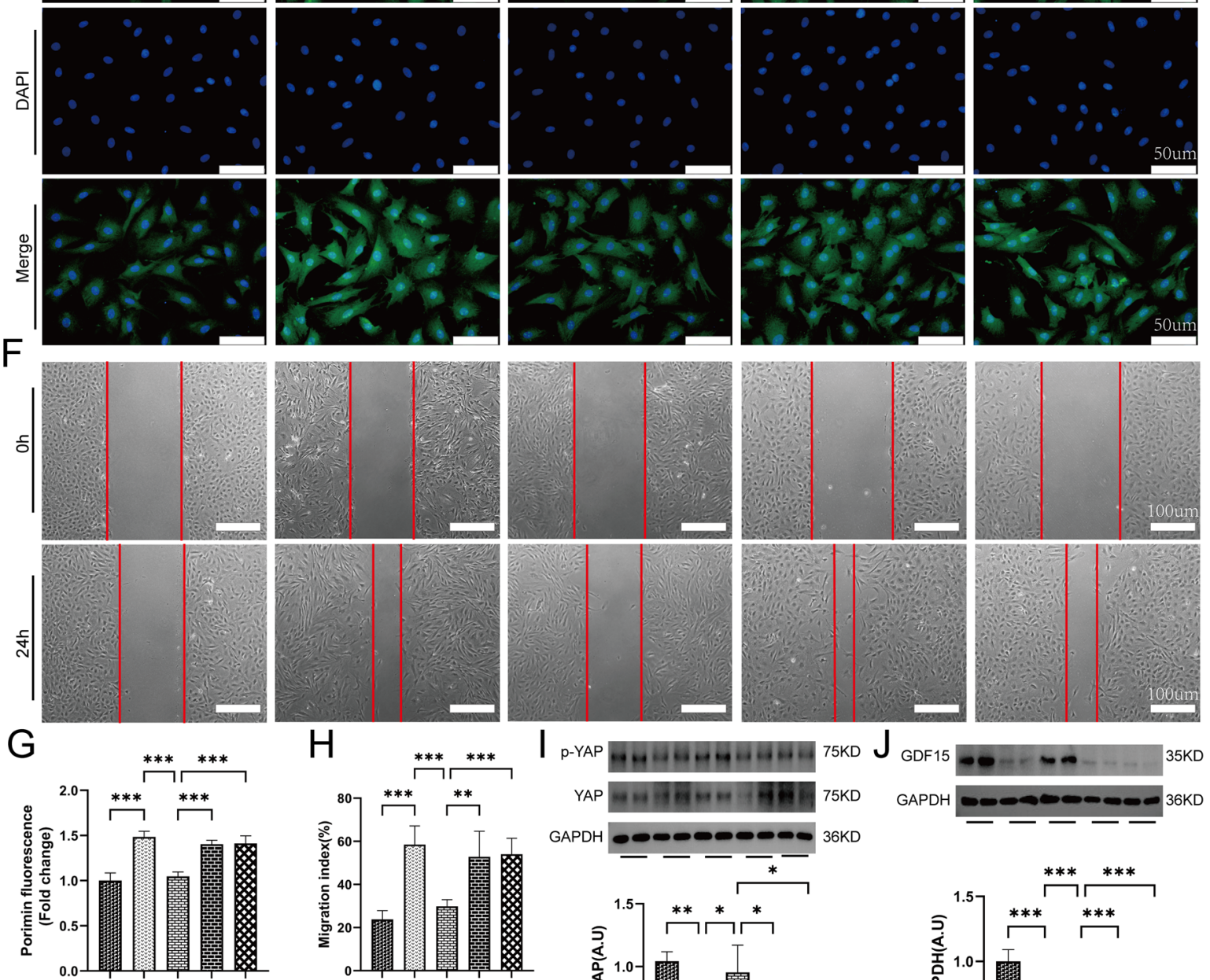

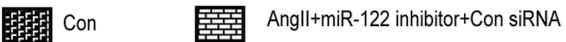

Angl|+NC Angl|+miR-122 inhibitor+Apelin siRNA

Angl|+miR-122 inhibitor+ELA siRNA
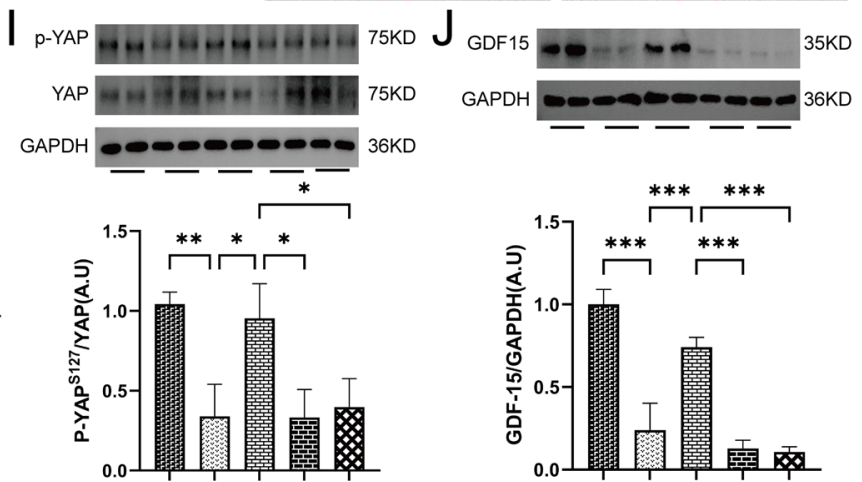
4Fig. 3 MiR-122 inhibitor significantly rescued Ang II-mediated increases in oncosis, cellular proliferation, and oxidative stress in cultured CFs via activating the ELA/apelin signaling. A, B The reverse transcription RT-PCR analysis revealed that miR-122 inhibitor rescued Ang II-meditated downregulation of apelin (A) and ELA (B) levels in Ang II-treated CFs, which were blocked by cotreatment with apelin siRNA (A) and ELA siRNA (B), respectively. C-H MiR-122 inhibitor suppressed Ang II-induced ROS generation (C, D) in cultured rat CFs, as well as expression of porimin $(\mathbf{E}, \mathbf{G})$ and cellular migration $(\mathbf{F}, \mathbf{H})$, which were abolished by apelin siRNA and ELA siRNA transfection, respectively. I-J Western blot analysis showed that silencing of apelin (I) and ELA ( $\mathbf{J})$ by siRNA abrogated miR122 inhibitor-mediated upregulation of p-YAP (I) and GDF15 (J). $\mathrm{CF}$, cardiac fibroblast; Ang II, angiotensin II; NC, negative control; ELA, elabela; ROS, reactive oxygen species; GDF15, growth differentiation factor 15; GAPDH, glyceraldehyde-3-phosphate dehydrogenase; YAP, yes-associated protein; A.U., arbitrary unit; R.E., relative expression. $n=5-6$ for each group except for $\mathbf{I}-\mathbf{J}$ where $n=3-4$. $* P<0.05, * * P<0.01, * * * P<0.001$

Assay Kit (Beyotime, Inc., China). Samples were diluted in $5 \times$ loading buffer, heated at $95^{\circ} \mathrm{C}$ for $5 \mathrm{~min}$, separated by SDS-PAGE, and transferred to ${ }^{\circledR}$ PVDF membrane (Millipore, MA, USA). Each membrane was pre-incubated in tris-buffered saline with Tween20 (TBST) containing 5\% nonfat milk for $1 \mathrm{~h}$ at room temperature and then incubated with specific primary antibodies against GDF15 (Santa Cruz, USA), porimin (Santa Cruz, USA), phosphorylated YAP (p-YAP; ABclonal, China), and YAP (Proteintech, USA) overnight at $4{ }^{\circ} \mathrm{C}$. After washing in TBST, the membranes were incubated with secondary antibodies conjugated to horseradish peroxidase, and immunoreactive bands were visualized using the enhanced chemiluminescence reagent (Immobilon ECL Ultra Western HRP Substrate, Millipore Sigma, USA). ImageJ was used to analyze protein band density.

\section{Reactive Oxygen Species and Malondialdehyde Assay}

The reactive oxygen species (ROS) level was detected by dihydroethidium (DHE) fluorescence staining (Beyotime, Shanghai, China) and ROS Assay Kit (Beyotime, Shanghai, China). Briefly, cells were incubated in DHE $(10 \mu \mathrm{M})$ for $30 \mathrm{~min}$ at $37^{\circ} \mathrm{C}$ and then observed by microscopy. Fluorescent images were captured with an Olympus IX51 microscope (Olympus, Tokyo) and fluorescence intensity of the probe was calculated with ImageJ software. Cells were incubated in the culture medium with $10 \mu \mathrm{M}$ 2,7-dichlorofluorescin diacetate at $37{ }^{\circ} \mathrm{C}$ for $30 \mathrm{~min}$. The activity of malondialdehyde (MDA) in CFs was determined using a commercial kit according to the manufacturer's instructions (Beyotime, Shanghai, China), then washed the cells with phosphate-buffered solution (PBS) three times, and suspended in $1 \mathrm{ml}$ PBS for flow cytometry analysis. The cells were quantified using a flow cytometer (BD Biosciences) and analyzed by the FlowJ software.

\section{Cell Transfection, Nitric Oxide, and Lactate Dehydrogenase Production Assay}

miR-122 inhibitor, specific small interfering RNAs (siRNA) for ELA, apelin and GDF15, and corresponding negative control (NC) siRNAs were purchased from GenePharma (Shanghai, China) and transiently transfected into CFs for $48 \mathrm{~h}$ at a concentration of $50 \mathrm{nM}$ using Lipofectamine 3000 reagent (Invitrogen, CA, USA). AngII $(1 \mu \mathrm{M})$ was then added to rat $\mathrm{CFs}$ for $24 \mathrm{~h}$. Nitric oxide (NO) and lactate dehydrogenase (LDH) production in CFs were assayed by using Total NO Assay Kit (Beyotime, Shanghai, China) and LDH kit (KGT02448, KeyGEN, China). The absorbance was measured using a full-wavelength multifunctional microplate reader JS-THERMO Varioskan Flash (Thermo, USA).

\section{Wound-Healing Assay and CCK8 Assay}

For wound healing assay, wounds were made by scratching with a $200-\mu \mathrm{l}$ pipette tip, and photographs were taken $0 \mathrm{~h}$ and $24 \mathrm{~h}$ after wounding. After $24 \mathrm{~h}$, rat CFs were washed with PBS and photographed to examine the migration of CFs into the middle line of the scratch. The scratch area was calculated using ImageJ software and the relative migration index was obtained from the ratio of cell migration distance at $0 \mathrm{~h}$ and $24 \mathrm{~h}$ with (migration distance at 0 -h migration distance at $24 \mathrm{~h}$ )/migration distance at $0 \mathrm{~h}$. CF migration was measured using a CCK8 kit (Beyotime, Shanghai, China) in accordance to the manufacturer's instructions. In brief, a total of 2000 cells in a volume of $100 \mu \mathrm{l}$ per well was cultured in a 96-well plate in medium containing $10 \%$ FBS. After treatment, the CCK-8 reagent $(10 \mu \mathrm{l})$ was added per well and incubated for $1 \mathrm{~h}$ at $37^{\circ} \mathrm{C}$ away from light before measuring the absorbance at $450 \mathrm{~nm}$ by a multifunctional microplate reader.

\section{Statistical Analysis}

All results were presented as the mean \pm standard deviation. Statistical analyses were performed using the Graphpad Prism 9.0.2 software and a value of $P<0.05$ was considered statistically significant. Student $t$ test was used for 2-sample comparisons; 1-way ANOVA with Student-Newman-Keuls post hoc test was performed to compare the data between multiple groups. 

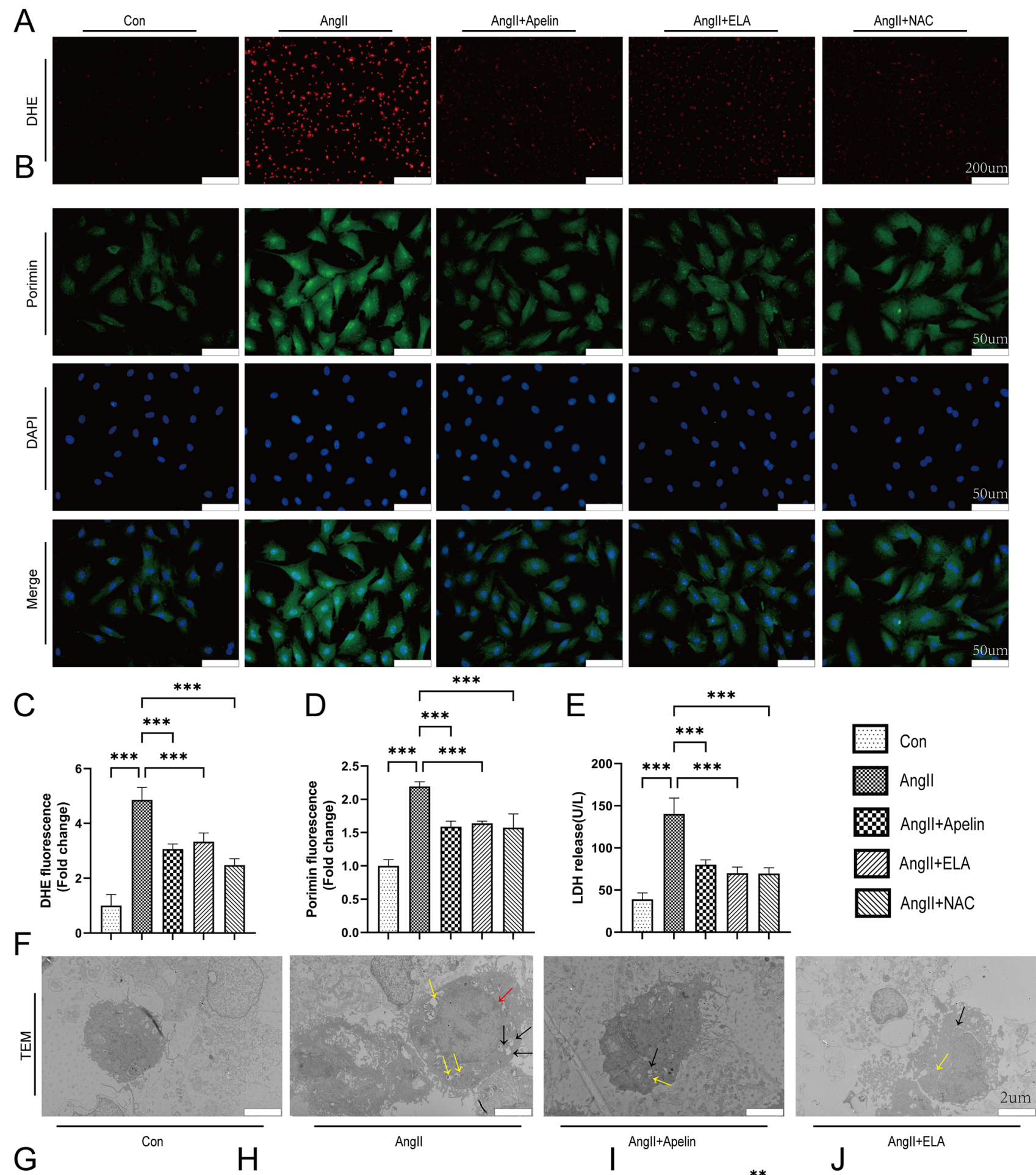

Angl|

Angll+Apelin

J Angll+ELA
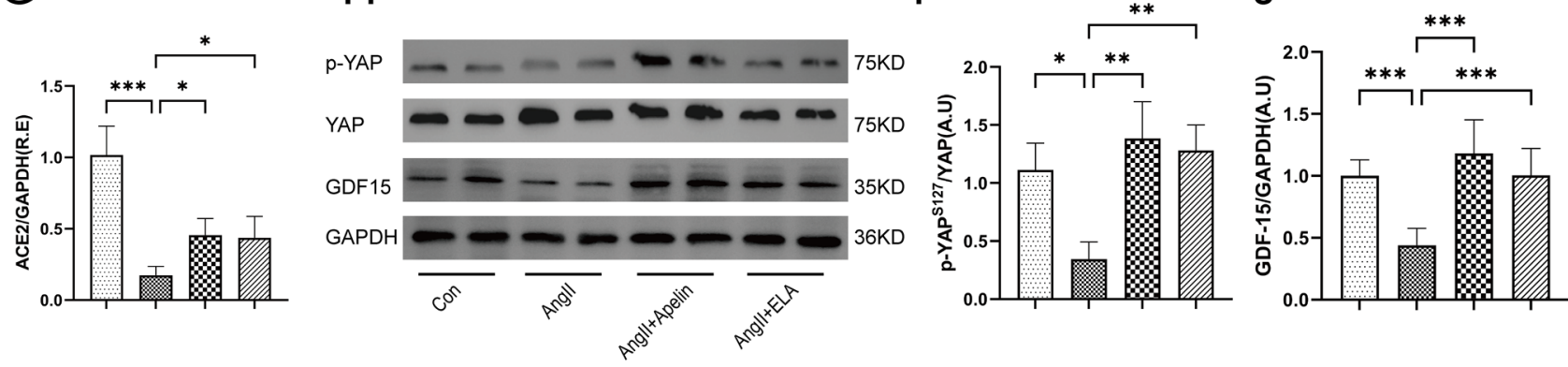
४Fig. 4 Treatment with apelin and ELA prevented Ang II-induced cellular oncosis and oxidative stress in CFs associated with upregulated levels of p-YAP and GDF15. A-D DHE staining and immunofluorescence revealed that pretreatemnet of apelin, ELA, and NAC prevented against Ang II-induced increases in ROS generation (A, C), and porimin expression (B, D). $\mathbf{E}$ The levels of $\mathrm{LDH}$ in each group. $\mathbf{F}$ Transmission electron microscopy showed that apelin and ELA attenuated Ang II-induced cellular oncosis-specific ultrastructural changes in CFs, characterized by swelling of organelles (yellow arrow) and nucleus (red arrow), and vacuolar degeneration of mitochondrial (black arrow). G-J Reverse transcription PCR (G) and western blot analysis $(\mathbf{H}-\mathbf{J})$ indicated that the ELA and apelin upregulated ACE2 mRNA expression $(\mathbf{G})$, as well as p-YAP $(\mathbf{H}, \mathbf{I})$ and protein expression of GDF15 $(\mathbf{H}, \mathbf{J})$ in Ang II-treated CFs. CF, cardiac fibroblast; Ang II, angiotensin II; ELA, elabela; ROS, reactive oxygen species; $\mathrm{LDH}$, lactate dehydrogenase; NAC, N-acetylcysteine; YAP, Yes-associated protein; GDF15, growth differentiation factor; GAPDH, glyceraldehyde-3-phosphate dehydrogenase. A.U., arbitrary unit; R.E., relative expression. $n=5-6$ for each group except for $\mathbf{H}-\mathbf{J}$ where $n=3$. $* * * P<0.001$

\section{Results}

MiR-122-5p Overexpression Promotes Myocardial Fibrosis, Cardiac Structural and Functional Injury in Hypertensive Rats via Downregulating the ELA/apelin-APJ Signaling.

We demonstrated that continuous infusion of Ang II for 4 weeks led to elevated SBP levels (Fig. 1A) and augmented cardiac expression of miR-122-5p (Fig. S1B) in rats, accompanied with reduced cardiac expression of apelin, APJ (Fig. 1B-E), and ELA (Fig. 1F). After administration of rAAV-miR122-5p, cardiac expression of miR-122-5p was further upregulated in hypertensive rats (Fig. S1B). In addition, miR-122-5p overexpression further decreased the expression of apelin, APJ, and ELA in hearts of hypertensive rats (Fig. 1B-F) However, administration of rAAV-miR-122-5p didn't affect the SBP levels (Fig. 1A). Importantly, the downregulated expression of ELA/apelin-APJ axis was linked with exacerbated cardiac tissue fibrosis in rAAV-miR-122-5ptreated hypertensive rats, as evidenced by increased cardiac collagen volume fraction (Fig. $1 \mathrm{G}-\mathrm{H}$ ) and elevated expression of pro-fibrotic factors, such as FN1, CTGF, and TGF- $\beta 1$ (Fig. 1I). Moreover, miR-122-5p overexpression aggravated myocardial ultrastructural injury in hypertensive rats (Fig. 2A), characterized by disorderly arranged myofilament, multiple myofilament breaks, and mitochondrial cristae rarefaction, leading to reduced left ventricular ejection fraction and fractional shortening (Fig. 2B-D). These results suggest that miR-122-5p overexpression promotes cardiac fibrosis, ultrastructural injury, and dysfunction in hypertensive rats through inactivating the ELA/ apelin-APJ signaling.

\section{MiR-122-5p Augments Oncosis in Hearts of Hypertensive Rats and Cultured Rat CFs with Decreased Levels of GDF15 and p-YAP}

Oncosis features the swelling of both the cells and the organelles specifically, together with increased membrane permeability [15], contributing to the progression of pressure overload-induced cardiac dysfunction [16].

Compared with rAAV-GFP, rAAV-miR-122-5p administration dramatically increased cardiac expression of porimin (Fig. 2E and $\mathrm{G}$ ), one oncosis-specific protein, in hypertensive rats. On the contrary, the cardiac expression of GDF15 (Fig. 2E and F) was suppressed in rAAV-miR$122-5 \mathrm{p}$-treated hypertensive rats. Based on the expression levels of porimin and GDF15, it seems plausible that miR$122-5$ p promotes cardiac oncosis through downregulating expression of GDF15 in hypertensive rats.

To further investigate the underlying mechanisms potentiating miR-122-5p-mediated cardiac pathological responses in hypertensive rats, we performed the in vitro experiments with primary cultured CFs and CMs. Consistent with the in vivo study, expression of miR-122-5p was upregulated in Ang II-treated CFs and CMs (Fig. S1C-S1D). Furthermore, both apelin and ELA mRNA expressions were decreased in Ang II-treated CFs (Fig. 3A and B), which were strikingly reversed by miR-122 inhibitor. It has been reported that, in an aerobic environment, cells are constantly generating ROS. However, excessive ROS under pathological conditions leads to oxidative stress, damage of intracellular molecules and organelles, and ultimately necrosis [17]. Notably, excessive production of ROS resulted in oncosislike cell death [18]. Importantly, the decrease of apelin and ELA expression in response to Ang II stimulation in CFs was linked with upregulated ROS (Fig. 3C, D), as well as augmented porimin expression (Fig. 3E and G) and cellular migration (Fig. 3F and $\mathrm{H}$ ). These changes were accompanied by an increase in mRNA levels of inflammatory biomarkers IL-1 $\beta$, IL-6, and MCP-1 (Fig. S3), and fibrotic biomarkers TGF- $\beta 1$, CTGF, and FN1 (Fig. S4).

Interestingly, miR-122 inhibitor abrogated Ang IIinduced downregulation of apelin and ELA in CFs (Fig. 3A, B), accompanied with decreasing levels of ROS (Fig. 3C, D), porimin (Fig. 3E and G), and cellular migration (Fig. 3F and $\mathrm{H}$ ). However, silencing of apelin and ELA partly abolished miR-122 inhibitor-mediated cellular protective effects (Fig. 3C-H), in parallel with downregulated levels of p-YAP and GDF15 protein expression. Our findings suggested that the ELA/apelin-APJ axis potentiated miR-122 inhibitor-mediated attenuation of oxidant damage, oncosis, and migration in cultured CFs, with GDF-15 and YAP being the downstream target. 
A
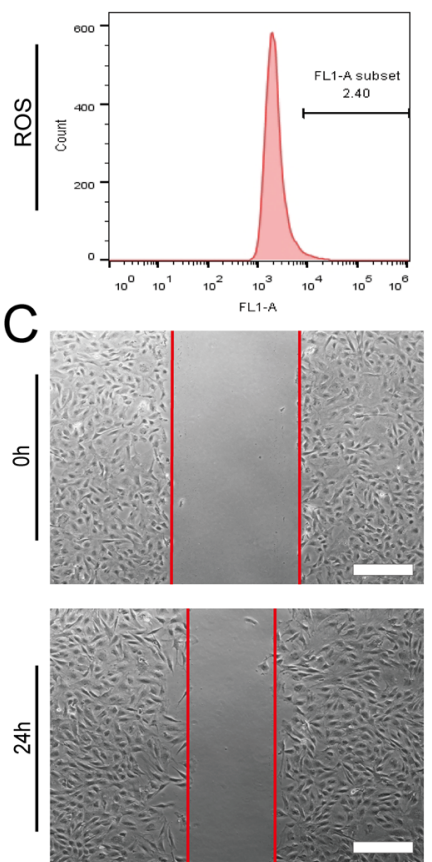
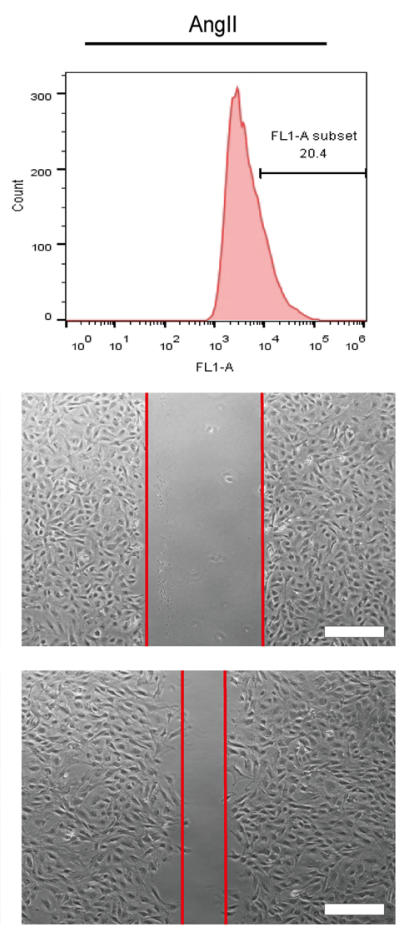
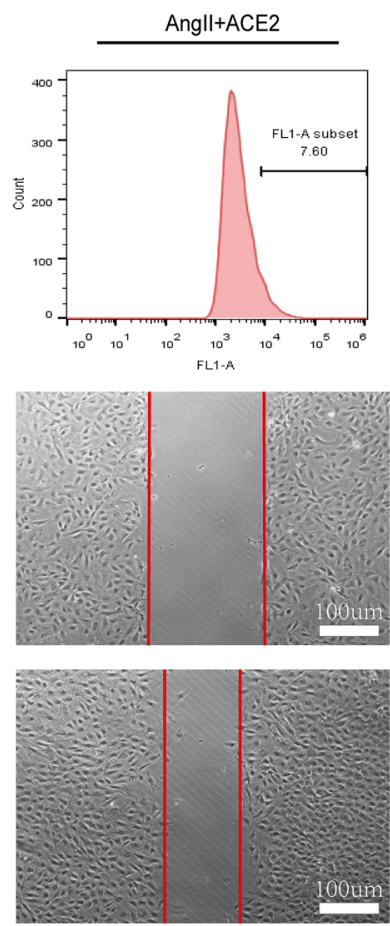

$\mathrm{B}$

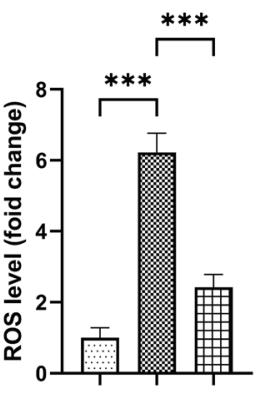

D

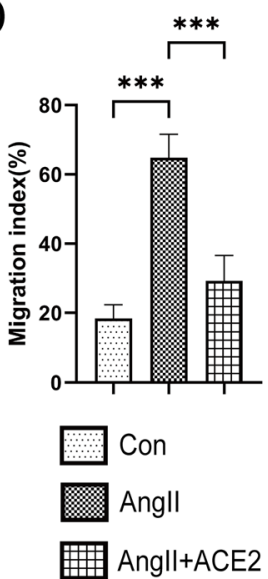

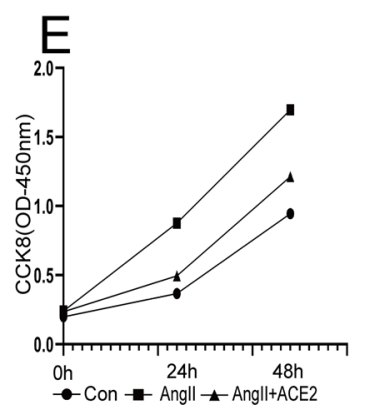

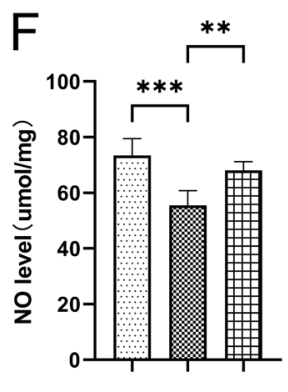

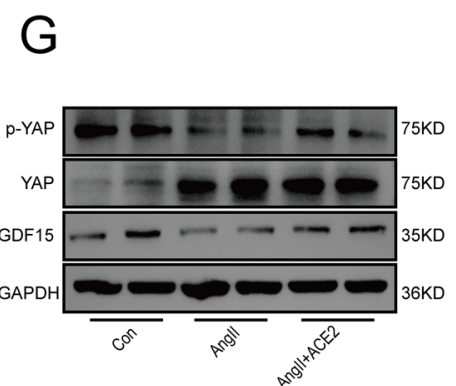

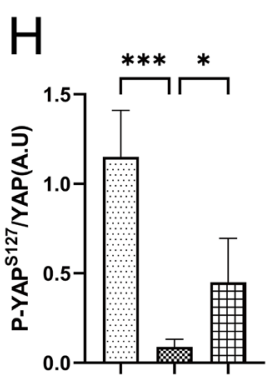

I

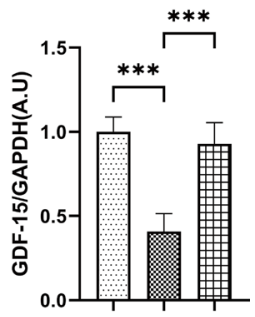

Fig. 5 ACE2 antagonized Ang II-induced augmentation of oxidative stress, migration, and proliferation in cultured CFs via activating the YAP-GDF15 signaling. A-E Flow cytometry assay (A, B), wound healing assay $(\mathbf{C}, \mathbf{D})$, and $\mathrm{CCK} 8(\mathbf{E})$ assay demonstrated that treatment with ACE2 alleviated Ang II-induced promotion of ROS production $(\mathbf{A}, \mathbf{B})$, cellular migration $(\mathbf{C}, \mathbf{D})$, and proliferation $(\mathbf{E})$ in cultured rat CFs. F Treatment with recombinant ACE2 rescued Ang II-mediated reduction in NO production in rat CFs. G-I The levels of p-YAP $(\mathbf{G}, \mathbf{H})$, YAP $(\mathbf{G}, \mathbf{H}), \operatorname{GDF} 15(\mathbf{H}, \mathbf{I})$ were detected by West-

\section{ELA/Apelin Prevents Against Ang II-Mediated Promotion of Oxidative Stress and Oncosis in CFs by Upregulating ACE2, GDF15, and p-YAP Levels}

We further investigated the molecular mechanisms that underlie the beneficial effects of ELA and apelin against Ang II-mediated oxidative stress, oncosis, and proliferation in CFs. DHE staining (Fig. 4A and C) and MDA assay (Fig. $\mathrm{S} 5 \mathrm{~A}$ ) revealed that treatment with ELA, apelin, and NAC ern blotting, and the results showed that expression of GDF15 (H, I) and the ratio of p-YAP/YAP $(\mathbf{G}, \mathbf{H})$ were upregulated by ACE2 in the Ang II-treated CFs. CF, cardiac fibroblast; ROS, reactive oxygen species; ACE2, angiotensin-converting enzyme; GDF15, growth differentiation factor; YAP, Yes-associated protein; GAPDH, glyceraldehyde-3-phosphate dehydrogenase; NO, nitric oxide; Ang II, angiotensin II; ELA, elabela; A.U., arbitrary unit; $n=5-6$ for each group except for $\mathbf{G}-\mathbf{I}$ where $n=4$. ${ }^{*} P<0.05, * * P<0.01$, $* * * P<0.001$

significantly restored Ang II-induced increases in cellular ROS generation (Fig. 4A and C); and lipid peroxidation in CFs (Fig. S5A), respectively. In addition, the increased porimin caused by Ang II was attenuated by ELA, apelin, and NAC, respectively (Fig. 4B and 4D).

It is well known that when cells swell due to the destruction of cell membrane integrity, cell contents extravasate and LDH is released outside the cell [19]. Our results illustrated that exogenous apelin, ELA, and NAC prevented Ang II-induced cell membrane damage, as evidenced by 
reduced LDH release (Fig. 4E) and NO production (Fig. $\mathrm{S} 5 \mathrm{~B}$ ) in CFs. Similar scenarios occurred with respect to cellular ultrastructure, as assessed by a transmission electron microscope, that treatment with apelin and ELA attenuated Ang II-induced organelles and nucleus swelling, and mitochondrial vacuolar degeneration (Fig. 4F). Notably, these beneficial effects caused by ELA and apelin were associated with upregulation of ACE2 (Fig. 4G), p-YAP (Fig. 4H and I), and GDF15 (Fig. 4H and J). Collectively, our data provide definitive evidence for a positive role of the ELA/apelin-APJ in Ang II-mediated cellular oncosis by upregulating ACE2, GDF15, and p-YAP.

\section{ACE2 Alleviates Ang II-Induced Increases in Oxidative Stress, Migration, Proliferation, and Oncosis in Rat CFs by Increasing GDF15 and $p$-YAP Levels}

To investigate the downstream signaling through which ACE2 counterbalances Ang II-induced cellular pathological changes, we conducted in vitro studies using rhACE2. The results revealed that exposure of Ang II resulted in marked increase in ROS generation (Fig. 5A, B), cellular migration (Fig. 5C, D), and proliferation (Fig. 5E) in cultured rat CFs, as well as significant reduction in NO production (Fig. 5F). Notably, these changes were reversed by co-treatment with rhACE2, in association with rescued levels of p-YAP (Figs. 5G, H) and GDF15 (Fig. 5G and I). We next tested the roles of GDF15 knockdown by siRNA in CFs. Transfection of GDF15 siRNA was confirmed to lower the expression of GDF15 protein (Fig. S6), accompanied with increased expression of porimin (Fig. S6). These results indicated that ACE2 plays a protective role by regulating the expression of GDF15 and p-YAP in Ang II-treated CFs.

Taken together, our data indicated that overexpression of miR-122-5p promoted fibrosis, inflammation, oncosis, and oxidant injury in hypertensive rats by suppressing the ELA/apelin signaling and their downstream ACE2-GDF15porimin pathway.

\section{Discussion}

Despite numerous efforts investigating the cause of hypertension and hypertensive heart disease, the precise underlying mechanisms are not completely clarified. Various miRNAs were reported to play critical roles in cardiac hypertrophy, fibrosis, and apoptosis, eventually fostering heart failure development [15]. It is well documented that the plasma miR-122 concentration was higher in essential hypertension patients than that in healthy individuals [15]. Although emerging evidences reveals that miR-122-5p plays an important role in the development of hypertension, cardiovascular fibrosis, and heart failure by targeting specific genes [8], the role and mechanism of miR-122-5p in the regulation of hypertensive heart disease remain unclear. To our knowledge, no prior study has addressed the role of miR$122-5 p$ in hypertensive heart disease. Here, we demonstrated that miR-122-5p overexpression contributed to exacerbation of cardiac fibrosis and dysfunction in hypertensive rats. Mechanistically, apelin and APJ were identified as the downstream target of miR-122-5p by using the publicly available bioinformatics tools Targetscan and miRWalk, which was further confirmed by that expression of apelin and APJ was significantly decreased in hearts of rAAV-miR-122-5ptreated hypertensive rats.

The ELA/apelin-APJ axis plays important roles in the cardiovascular homeostasis and development. Its agonists are being considered therapeutic agents to modulate cardiovascular homeostasis in adults. The new endogenous peptide ligand for APJ, ELA, has been expected to become a biomarker and drug of cardiovascular diseases [13]. Circulating ELA and apelin levels were decreased in patients with essential hypertension and general hypertension [13]. The ELA/ apelin-APJ axis is widely expressed in cardiovascular system, and exerts a variety of important functions, including vascular tone regulation, angiogenesis, and heart morphogenesis [13], suggesting that determination and modulation of the activity of ELA/apelin-APJ axis have predictive and therapeutic value. In the current study, rAAV-miR-122-5p treatment further led to downregulation of ELA in hearts of hypertensive rats. In contrast, miR-122 inhibitor strikingly restored Ang II-mediated reduction of ELA in cultured rat CFs. Here, we demonstrated for the first time that miR-122-5p targeted the ELA/apelin-APJ axis to facilitate hypertensive cardiac fibrosis and dysfunction.

It is well established that GDF15, which can be induced in hypertrophic and dilated cardiomyopathy after volume overload, functions as a biomarker of response to treatment and prognosis in cardiovascular diseases [3]. In cultured primary CFs and cardiomyocytes, treatment with Ang II significantly downregulated the expression of GDF15, accompanied by enhancement of apoptosis and inflammation [20]. Consistent with previous study, we found that expression of GDF15 was decreased in both the hearts of hypertensive rats and Ang II-treated CFs, which was further reduced by overexpression of miR-122-5p while being upregulated by ELA and apelin, respectively.

As is well known, apelin is a positive regulator of ACE2 in failing hearts [11], and ACE2 has been identified as an important RAS regulator capable of mitigating the deleterious actions mediated by Ang II [21]. We revealed here that administration of ACE2 reversed Ang II-mediated downregulation of GDF15 and p-YAP in cultured rat CFs, along with alleviated cellular proliferation and oxidative 
stress. Furthermore, silencing of GDF15 by siRNA abolished ACE2-mediated protective effects against Ang II, confirming GDF15 was a downstream molecule of ACE2. It has been reported that YAP mediates Ang II-induced vascular smooth muscle cell phenotypic modulation and hypertensive vascular remodeling [22, 23], and blockade of fibroblast YAP attenuates cardiac fibrosis and dysfunction [7]. Our study confirmed that the ratio of pYAP/YAP was downregulated in response to Ang II stimulation, which was reversed by miR-122 inhibitor, ELA, and apelin, respectively. These data provided important evidence that the ELA/apelin-ACE2 signaling could control pathological cardiac remodeling via modulating YAP phosphorylation. However, further study based on YAP inhibitor is warranted to confirm the effects and precise molecular mechanisms of YAP in CFs treated with Ang II.

Our data noted above proved for the first time that administration of miR-122-5p exacerbated Ang IImediated promotion of myocardial fibrosis and dysfunction by suppressing the ELA/apelin-APJ axis and the ACE2-GDF15/YAP pathway. However, differing from our conclusions, Ramu et al. reported that elevated level of GDF15 is associated with increased concentration of Ang II in hypertensive patients with type 2 diabetes, and GDF15 mRNA expression was significantly elevated in Ang-II-treated H9C2 cells [4]. Previous literature also showed that Ang II-induced GDF15 expression conferred protection against Ang II-mediated apoptosis in cultured rats' cardiomyocytes [24]. The findings are contradicting, which might be explained in part by different animal models and doses of Ang II used in each study.

Death of numerous cardiomyocytes results in reparative fibrosis; therefore, breaking the fibrosis-cell death axis could suppress pathological cardiac regression and remodeling [25]. Notably, several other forms of cell death have been discovered highlighting that a cell can die via a number of differing pathways [15]. It has been reported that cell loss, mainly caused by oncosis and autophagy, contributes significantly to the progression of pressure overload-induced cardiac dysfunction [16]. However, the underlying mechanisms remain largely unknown. In this study, we found that Ang II promoted oncosis in vivo and in vitro, as evidenced by the high expression of porimin (a specific marker of oncosis). In addition, the expression of porimin was further increased in hearts of rAAV-miR122-5ptreated hypertensive rats, and the Ang II-induced elevated expression of porimin was suppressed in cultured rat CFs administrated with miR-122 inhibitor, ELA, and apelin, respectively. It is worth noting that knockdown of GDF15 in CFs partially abrogated ACE2-mediated regression on expression of porimin. These data strongly suggest that miR-122-5p promotes oncosis in hearts of hypertensive rats via modulating the ELA/apelin-APJ pathway and the ACE2-GDF15-porimin signaling.

\section{Conclusion}

Collectively, in the present study, we revealed that miR$122-5 p$ overexpression results in downregulation of cardiac apelin and ELA in hypertensive rats, leading to augmented cardiac fibrosis, structural injury, and dysfunction. To our knowledge, this study for the first time present that miR-122-5p promotes oncosis and cardiac remodeling in hypertensive rats by modulating the ELA/apelin-APJ axis and the ACE2-GDF15-porimin signaling pathways. Our data reveal a novel mechanism for miR-122-5p in the exacerbation of Ang II-mediated promotion of cardiac fibrosis and dysfunction in hypertensive rats. In addition, our findings provide a novel conception which manipulation of the miR-122-5p will offer exciting avenues for the prevention and therapy of hypertension and hypertensive cardiac disease.

Supplementary Information The online version contains supplementary material available at https://doi.org/10.1007/s12265-022-10214-3.

Author Contribution Jiuchang Zhong, Haiyan Jin, Jiawei Song, and Zhenzhou Zhang designed the study and wrote the protocols; Jiawei Song, Zhenzhou Zhang, Zhaojie Dong, Xinming Liu, Ying Liu, Xueting $\mathrm{Li}$, and Yingle $\mathrm{Xu}$ prepared the materials and performed the experiments; Ying Guo, Ning Wang, Miwen Zhang, and Yihang Chen collected and analyzed the data; Jiawei Song and Zhenzhou Zhang wrote the first draft of the manuscript; Jiuchang Zhong and Haiyan Jin revised the manuscript. All the authors read and approved the final manuscript.

Funding This study was supported by the General Program and the National Major Research Plan Training Program of the National Natural Science Foundation of China (No. 92168117; 81770253; 91849111; 81370362), Natural Science Foundation of Beijing (No. 7222068), Shanghai Sailing Program (No. 20YF1444100), and Beijing Postdoctoral Research Foundation.

Data Availability The datasets generated and/or analyzed during the current study are available from the corresponding author on reasonable request.

\section{Declarations}

Conflict of Interest The authors declare no competing interests.

Ethical Approval No human studies were carried out by the authors for this article. This study was performed in line with the principles of the Declaration of Helsinki. All animal procedures were performed in accordance with the Guide for the Care and Use of Laboratory Animals published by the Animal Research Ethics Committee of Beijing Chaoyang Hospital affiliated to Capital Medical University and Animal Research Ethics Committee at Shanghai Jiao Tong University School of Medicine. 


\section{References}

1. Wang, Z., Chen, Z., Zhang, L., Wang, X., Hao, G., Zhang, Z., Shao, L., Tian, Y., Dong, Y., Zheng, C., Wang, J., Zhu, M., Weintraub, W. S., Gao, R., \& China Hypertension Survey, I. (2018). Status of hypertension in China: Results from the China Hypertension Survey, 2012-2015. Circulation., 137, 23442356. https://doi.org/10.1161/CIRCULATIONAHA.117.032380

2. Gonzalez, A., Ravassa, S., Lopez, B., Moreno, M. U., Beaumont, J., San, Jose G., Querejeta, R., Bayes-Genis, A., \& Diez, J. (2018). Myocardial remodeling in hypertension. Hypertension., 72, 549-558. https://doi.org/10.1161/HYPERTENSI ONAHA.118.11125

3. Arkoumani, M., Papadopoulou-Marketou, N., Nicolaides, N. C., Kanaka-Gantenbein, C., Tentolouris, N., \& Papassotiriou, I. (2020). The clinical impact of growth differentiation factor- 15 in heart disease: A 2019 update. Crit. Rev. Clin. Lab. Sci., 57, 114-125. https://doi.org/10.1080/10408363.2019.1678565

4. Adela, R., Mohammed, S. A., Kanwal, A., Vishwakarma, G., Chander Reddy, P. N., \& Banerjee, S. K. (2016). Elevated levels of GDF-15 is associated with increased angiotensin II in hypertensive patients with type 2 diabetes. Per. Med., 13, 325-336. https://doi.org/10.2217/pme-2016-0030

5. Wang, J., Liu, S., Heallen, T., \& Martin, J. F. (2018). The Hippo pathway in the heart: Pivotal roles in development, disease, and regeneration. Nat. Rev. Cardiol., 15, 672-684. https://doi.org/ 10.1038/s41569-018-0063-3

6. Niu, L., Jia, Y., Wu, M., Liu, H., Feng, Y., Hu, Y., Zhang, X., Gao, D., Xu, F., \& Huang, G. (2020). Matrix stiffness controls cardiac fibroblast activation through regulating YAP via AT1 R. J. Cell. Physiol., 235, 8345-8357. https://doi.org/10.1002/jcp.29678

7. Francisco, J., Zhang, Y., Jeong, J. I., Mizushima, W., Ikeda, S., Ivessa, A., Oka, S., Zhai, P., Tallquist, M. D., \& Del Re, D. P. (2020). Blockade of fibroblast YAP attenuates cardiac fibrosis and dysfunction through MRTF-A inhibition. JACC Basic Transl Sci., 5, 931-945. https://doi.org/10.1016/j.jacbts.2020.07.009

8. Liu, Y., Song, J. W., Lin, J. Y., Miao, R., \& Zhong, J. C. (2020). Roles of microrna-122 in cardiovascular fibrosis and related diseases. Cardiovasc. Toxicol., 20, 463-473. https://doi.org/10.1007/ s12012-020-09603-4

9. Weber, G. J., Purkayastha, B., Ren, L., Pushpakumar, S., \& Sen, U. (2018). Hypertension exaggerates renovascular resistance via miR-122-associated stress response in aging. J. Hypertens., 36, 2226-2236. https://doi.org/10.1097/HJH.0000000000001770

10. Song, J. J., Yang, M., Liu, Y., Song, J. W., Wang, J., Chi, H. J., Liu, X. Y., Zuo, K., Yang, X. C., \& Zhong, J. C. (2020). MicroRNA-122 aggravates angiotensin II-mediated apoptosis and autophagy imbalance in rat aortic adventitial fibroblasts via the modulation of SIRT6-elabela-ACE2 signaling. Eur. J. Pharmacol., 883, 173374. https://doi.org/10.1016/j.ejphar.2020.173374

11 Sato, T., Suzuki, T., Watanabe, H., Kadowaki, A., Fukamizu, A., Liu, P. P., Kimura, A., Ito, H., Penninger, J. M., Imai, Y., \& Kuba, $\mathrm{K}$. (2013). Apelin is a positive regulator of ACE2 in failing hearts. J. Clin. Invest., 123, 5203-11. https://doi.org/10.1172/JCI69608

12. Xu, R., Zhang, Z. Z., Chen, L. J., Yu, H. M., Guo, S. J., Xu, Y. L., Oudit, G. Y., Zhang, Y., Chang, Q., Song, B., Chen, D. R., Zhu, D. L., \& Zhong, J. C. (2016). Ascending aortic adventitial remodeling and fibrosis are ameliorated with Apelin-13 in rats after TAC via suppression of the miRNA-122 and LGR4-betacatenin signaling. Peptides., 86, 85-94. https://doi.org/10.1016/j. peptides.2016.10.005

13. Song J.W., Tang J.Q., Zhang Z.Z., Liu Y.\&Zhong J.C. (2021) Targeting the elabela/apelin-apelin receptor axis as a novel therapeutic approach for hypertension. Chin. Med. J. (Engl.). https://doi.org/10.1097/cm9.0000000000001766

14. Zhang, Z. Z., Wang, W., Jin, H. Y., Chen, X., Cheng, Y. W., Xu, Y. L., Song, B., Penninger, J. M., Oudit, G. Y., \& Zhong, J. C. (2017). Apelin Is a negative regulator of angiotensin II-mediated adverse myocardial remodeling and dysfunction. Hypertension., 70,1165-1175. https://doi.org/10.1161/HYPERTENSIONAHA. 117.10156

15 D'Arcy, M. S. (2019). Cell death: A review of the major forms of apoptosis, necrosis and autophagy. Cell Biol. Int., 43, 582-592. https://doi.org/10.1002/cbin.11137

16 Hein, S., Arnon, E., Kostin, S., Schonburg, M., Elsasser, A., Polyakova, V., Bauer, E. P., Klovekorn, W. P., \& Schaper, J. (2003). Progression from compensated hypertrophy to failure in the pressure-overloaded human heart: Structural deterioration and compensatory mechanisms. Circulation., 107, 984-91. https:// doi.org/10.1161/01.cir.0000051865.66123.b7

17 Ricci, M. S., \& Zong, W. X. (2006). Chemotherapeutic approaches for targeting cell death pathways. Oncologist., 11, 342-57. https:// doi.org/10.1634/theoncologist.11-4-342

18. Du, J. H., Zhang, H. D., Ma, Z. J., \& Ji, K. M. (2010). Artesunate induces oncosis-like cell death in vitro and has antitumor activity against pancreatic cancer xenografts in vivo. Cancer Chemother. Pharmacol., 65, 895-902. https://doi.org/10.1007/ s00280-009-1095-5

19 Chu, J., Ji, H., Lu, M., Li, Z., Qiao, X., Sun, B., Zhang, W., \& Xue, D. (2013). Proteomic analysis of apoptotic and oncotic pancreatic acinar AR42J cells treated with caerulein. Mol. Cell. Biochem., 382, 1-17. https://doi.org/10.1007/s11010-013-1603-0

20. Chen, B., Lu, D., Fu, Y., Zhang, J., Huang, X., Cao, S., Xu, D., Bin, J., Kitakaze, M., Huang, Q., \& Liao, Y. (2014). Olmesartan prevents cardiac rupture in mice with myocardial infarction by modulating growth differentiation factor 15 and p53. Br. J. Pharmacol., 171, 3741-53. https://doi.org/10.1111/bph.12736

21. Patel, V. B., Zhong, J. C., Grant, M. B., \& Oudit, G. Y. (2016). Role of the ACE2/angiotensin 1-7 axis of the renin-angiotensin system in heart failure. Circ. Res., 118, 1313-26. https://doi.org/ 10.1161/CIRCRESAHA.116.307708

22 Wu, P., Liu, Z., Zhao, T., Xia, F., Gong, L., Zheng, Z., Chen, Z., Yang, T., \& Duan, Q. (2019). Lovastatin attenuates angiotensin II induced cardiovascular fibrosis through the suppression of YAP/ TAZ signaling. Biochem. Biophys. Res. Commun., 512, 736-741. https://doi.org/10.1016/j.bbrc.2019.03.158

23. Lin, M., Yuan, W., Su, Z., Lin, C., Huang, T., Chen, Y., \& Wang, J. (2018). Yes-associated protein mediates angiotensin II-induced vascular smooth muscle cell phenotypic modulation and hypertensive vascular remodelling. Cell Prolif., 51, e12517. https://doi. org/10.1111/cpr.12517

24. Heger, J., Schiegnitz, E., von Waldthausen, D., Anwar, M. M., Piper, H. M., \& Euler, G. (2010). Growth differentiation factor 15 acts anti-apoptotic and pro-hypertrophic in adult cardiomyocytes. J. Cell. Physiol., 224, 120-6. https://doi.org/10.1002/jcp.22102

25 Piek, A., de Boer, R. A., \& Sillje, H. H. (2016). The fibrosis-cell death axis in heart failure. Heart Fail. Rev., 21, 199-211. https:// doi.org/10.1007/s10741-016-9536-9

Publisher's Note Springer Nature remains neutral with regard to jurisdictional claims in published maps and institutional affiliations. 